\title{
Development and Validation of a Scoring System for Differential Diagnosis of Tuberculosis and Metastatic Tumor in the Spine
}

\author{
Shuang Cao ${ }^{1,2, *}$ \\ Xin Gao ${ }^{l, *}$ \\ Guangjian Bai (D) ${ }^{1, *}$ \\ Baoquan Xin ${ }^{1, *}$ \\ Tao Wang' \\ Jiashi Cao' \\ Kai Lv' \\ Chengzhang Zhu' \\ Xiangzhi $\mathrm{Ni}^{\text {I }}$ \\ Weiwei Zou ${ }^{3}$ \\ Yejin Zhou ${ }^{2}$ \\ Jianru Xiao' \\ Tielong Liu' \\ 'Orthopedic Oncology Center, \\ Department of Orthopedics, Changzheng \\ Hospital, Second Military Medical \\ University, Shanghai, People's Republic of \\ China; ${ }^{2}$ Department of Orthopedics, \\ Third Affiliated Hospital of Anhui Medical \\ University, Hefei, Anhui Province, \\ People's Republic of China; ${ }^{3}$ Department \\ of Radiology, Changzheng Hospital, \\ Second Military Medical University, \\ Shanghai, People's Republic of China \\ *These authors contributed equally to \\ this work
}

Correspondence: Tielong Liu; Jianru Xiao Orthopedic Oncology Center, Department of Orthopaedics,

Changzheng Hospital, No. 4I5 Fengyang

Road, Huangpu District, Shanghai,

People's Republic of China

Tel +86-2I-8I886843

Fax +86-2I-63520020

Email liutielongvip@I63.com;

xiaojianruvvip@163.com
This article was published in the following Dove Press journal: Infection and Drug Resistance

Purpose: Spinal tuberculosis (TB) and metastatic tumor (MT) are common diseases with similar manifestations. Although pathological evaluation is the gold standard to confirm diagnosis, performing biopsies in all patients is not feasible. This study is aimed to create a scoring system to facilitate the differential diagnosis of spinal TB and MT before invasive procedures. Methods: Altogether, 447 patients with spinal TB $(n=198)$ and MT $(n=249)$ were retrospectively analyzed. Patients were randomly assigned at 2:1 ratio to a training cohort and a validation cohort. Clinical, laboratory, and radiological diagnostic factors were identified by $\chi^{2}$ and multiple logistic regression analyses. The scoring system was then established based on the identified independent diagnostic factors scored by regression coefficient $\beta$ value, with the cut-off value being determined by ROC curve. The sensitivity and specificity of the system was calculated by comparing the predicted diagnosis with their actual pathological diagnosis.

Results: This scoring system was composed of 5 items: pain worsens at night ( 0 or 2 points), CRP value ( 0 or 3 points), tumor marker values ( 0 or 2 points), skip lesions ( 0 or 3 points), and intervertebral space destruction ( 0 or 3 points). Patients scoring higher than 7.5 could be diagnosed as spinal TB, otherwise, MT. According to the internal validation, the sensitivity and specificity of the system were $87.9 \%$ and $91.6 \%$, respectively.

Conclusion: This study established and validated a scoring system which could be used to differentiate spinal TB from MT, thus helping clinicians in quick and accurate differential diagnosis.

Keywords: scoring system, differential diagnosis, spine, tuberculosis, metastatic tumor

\section{Introduction}

Tuberculosis (TB) and metastatic tumor (MT) are frequently diagnosed lesions in the spine. The incidence of spinal TB ranks top in TB of bones and joints, accounting for approximately $50 \%$ of all skeletal TB cases. ${ }^{1}$ The spine is also the most common site of osseous metastatic disease, with about $60 \%$ of the bone MT being spinal MT. ${ }^{2}$ Although spinal TB and MT are two different disease entities, both showed vertebral bone destruction and local mass on the imaging examination. ${ }^{3}$ Meanwhile, nonspecific back pain represents the most common symptom in both diseases. In later stages, both have the potential to cause spinal cord compression, leading to neurological sequelae including paraplegia. ${ }^{1,4}$ Given the similar manifestations, differential diagnosis of the two diseases constitutes a clinical challenge. ${ }^{3,5,6}$

Although pathological examination is the gold standard for differential diagnosis of spinal TB and MT, sometimes patients with acute symptomatic spinal cord compression 
require prompt surgical decompression with no time to receive a biopsy in advance. Surgeons usually make the differential diagnosis based on one or some certain features of the two diseases, such as the presence or absence of intervertebral space destruction, the level(s) of erythrocyte sedimentation rate (ESR) and/or C-reactive protein (CRP), rather than a systematic scoring system. ${ }^{5}$ Although these features are effective in most cases, it would be very difficult to be distinguished for atypical cases, such as noncontiguous multisegmental spinal TB with no intervertebral disc involvement. ${ }^{7}$ Actually, due to atypical presentations, both cases of spinal TB misdiagnosed as MT, ${ }^{8-11,6}$ and spinal MT cases mistaken for $\mathrm{TB}^{12-14}$ were not rare in clinical practice. The missed diagnosis often led to inappropriate treatment, especially regarding the surgical options, resulting in devastating consequences. $^{10,12}$ Therefore, systematic guidance on differential diagnosis of spinal TB and MT is of great importance for clinicians.

In this study, the clinical, laboratory, and radiological data of 447 patients with spinal TB or MT were systemically analyzed to develop and validate a novel and practical scoring system for the differential diagnosis between TB and MT in the spine.

\section{Patients and Methods}

\section{Patient Selection}

Included in this retrospective study were 447 consecutive patients with spinal TB $(n=198)$ or MT $(n=249)$ who received surgical treatment in our center between July 2014 and July 2018. Patient selection was restricted to fulfill the following inclusive criteria: (1) the final diagnosis of spinal TB or MT was confirmed by two pathologists independently; and (2) patients developing symptomatic spinal cord compression were planned to receive surgical treatment. In addition, patients diagnosed with nontuberculous infection or primary tumor in the spine were excluded from this study. The flow diagram for the study design is shown in Figure 1. Using a permuted randomization protocol, both TB and MT cases were randomly assigned at a 2:1 ratio to a training cohort (132 TB cases and 166 MT cases) for univariate/multivariate analyses and scoring system development, and a validation cohort (66 TB cases and 83 MT cases) for validation of the proposed system. All procedures performed in this study were approved by the Ethics Committee of the Second Affiliated Hospital of Second Military Medical University. We declare that the written informed consent for publication of their details was

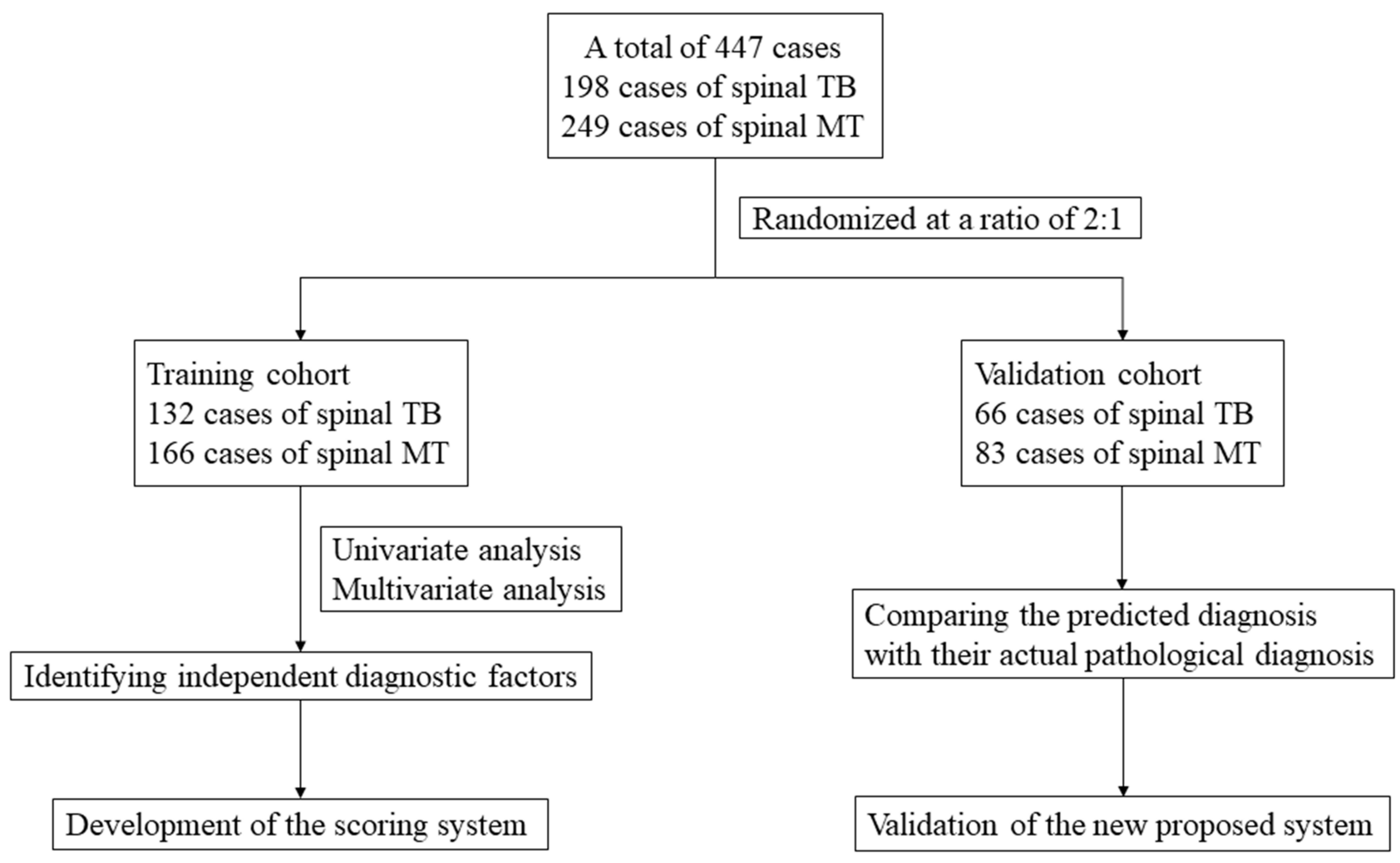

Figure I Flow diagram for study design. 
obtained from all patients and the study has been conducted in accordance with the Declaration of Helsinki.

\section{Data Collection}

The clinical, laboratory, radiological, and pathological data of all included patients were reviewed by two researchers. The clinical data included gender, age, tumor history, the presence or absence of low fever, night sweats, and pain worsens at night. Laboratory data included white blood cell (WBC) count, levels of ESR, CRP, and tumor markers (AFP, PSA, CEA, CA199, CA125, CA15-3, CEA72-4). These data were dichotomized according to their normal ranges. Radiological data (X-ray, CT, and MRI) included skip lesions, intervertebral space destruction, paraspinal abscess, and vertebral appendices involvement. The imaging data were interpreted by two radiologists independently. Dichotomized classification was carried out according to the presence or absence of these parameters. Disagreement was resolved by a third opinion.

\section{Development of Scoring System}

Statistical analyses were performed using SPSS Statistics version 22.0 (IBM Corporation, Armonk, NY, USA). All the above-mentioned clinical, laboratory, and radiological parameters in the training cohort were entered for univariate analysis using $\chi^{2}$ test. All variables significant at $\mathrm{p}<0.05$ in the univariate analysis were subjected to multivariate analysis using multiple logistic regression analysis. Variables in the multivariate analysis with $\mathrm{p}$ values of $<0.1$ were recognized as independent diagnostic factors.

The independent diagnostic factors were regarded as the items in the scoring system. The score of each item was defined based on their regression coefficient $\beta$ value in multiple logistic regression model, with the values being rounded off to the nearest integer. The optimal cut-off value of the scoring system was determined by the receiver operating characteristic (ROC) curve corresponding to the point on the curve with the largest area under ROC.

\section{Validation of Scoring System}

In validationthe cohort, the total score of each patient was calculated based on the new proposed system, and then the predicted diagnosis was determined according to the cutoff value. By comparing between the predicted diagnosis and their actual pathological diagnosis, the sensitivity and specificity of this scoring system were obtained.
Table I Univariate Analyses on Potential Diagnostic Factors for Differentiation Between Spinal TB and MT (Training Cohort)

\begin{tabular}{|c|c|c|c|}
\hline Factors & $\begin{array}{l}\text { Spinal TB } \\
(n=132)\end{array}$ & $\begin{array}{l}\text { Spinal MT } \\
(n=166)\end{array}$ & $\mathbf{P}$ \\
\hline \multicolumn{4}{|l|}{ Clinical factors } \\
\hline Gender (male) & $85(64.4 \%)$ & $104(62.7 \%)$ & 0.756 \\
\hline Age $(<55$ y) & $63(47.7 \%)$ & $53(31.9 \%)$ & 0.005 \\
\hline Without tumor history & $129(97.7 \%)$ & $15(9.0 \%)$ & $<0.001$ \\
\hline Low fever and sweating & 42 (3I.8\%) & I $(0.6 \%)$ & $<0.001$ \\
\hline No pain worsens at night & 105 (79.5\%) & 107 (64.5\%) & 0.004 \\
\hline \multicolumn{4}{|l|}{ Laboratory factors } \\
\hline High WBC value & $10(7.6 \%)$ & $22(13.3 \%)$ & 0.116 \\
\hline High ESR value & $106(80.3 \%)$ & 118 (7I. I\%) & 0.067 \\
\hline High CRP value & 73 (55.3\%) & $64(38.6 \%)$ & 0.004 \\
\hline $\begin{array}{l}\text { Normal tumor marker } \\
\text { values }\end{array}$ & $104(78.8 \%)$ & $48(28.9 \%)$ & $<0.001$ \\
\hline \multicolumn{4}{|l|}{ Radiological factors } \\
\hline Isolated lesions & $126(95.5 \%)$ & $94(56.6 \%)$ & $<0.001$ \\
\hline $\begin{array}{l}\text { Intervertebral space } \\
\text { destruction }\end{array}$ & II3 (85.6\%) & $9(5.4 \%)$ & $<0.00$ I \\
\hline Paraspinal abscess & $45(34.1 \%)$ & $0(0.0 \%)$ & $<0.00$ I \\
\hline $\begin{array}{l}\text { No vertebral appendices } \\
\text { involvement }\end{array}$ & $47(35.6 \%)$ & $23(13.9 \%)$ & $<0.001$ \\
\hline
\end{tabular}

Note: Bold values indicate statistical significance $P<0.05$.

\section{Results}

\section{Patient Characteristics}

The total cohort was composed of 264 males and 183 females, with a mean age of 55.5 years (median, 55; range, 17-80). The mean age of the patients with spinal TB and MT were 51.9 and 56.4 years, respectively. Low fever and night sweats were common symptoms in $57(28.8 \%)$ TB patients, while pain worsens at night was observed in 88 (35.3\%) MT patients. Of the 249 patients with spinal MT, $220(88.4 \%)$ patients had a tumor history. ESR and CRP were elevated in 150 (75.8\%) and 115 (58.1\%) TB patients, respectively. Elevation of tumor markers was detected in $172(69.1 \%)$ MT patients. Intervertebral space destruction was observed in 157 (79.3\%) patients with spinal TB, and skip lesions were found in 105 (42.2\%) patients with spinal MT.

\section{Diagnostic Factors for Differentiating Spinal TB from MT}

In the training cohort, the results of univariate analysis to identify the potential diagnostic factors for differentiation between spinal TB and MT are shown in Table 1. Age $<55$ years, without tumor history, low fever, and night sweats, 
Table 2 Multiple Logistic Regression Analysis on Diagnostic Factors for Differentiation Between Spinal TB and MT (Training Cohort)

\begin{tabular}{|l|l|l|l|}
\hline Factors & $\mathbf{P}$ & $\boldsymbol{\beta}$ & OR $(\mathbf{9 0 \%} \mathbf{C l})$ \\
\hline Age $(<55$ y) & 0.105 & - & - \\
Without tumor history & 0.991 & - & - \\
Low fever and sweating & 0.993 & - & - \\
No pain worsens at night & $\mathbf{0 . 0 7 0}$ & 2.17 & $8.79(1.22-63.30)$ \\
High CRP value & $\mathbf{0 . 0 8 7}$ & 3.27 & $26.30(1.13-611.57)$ \\
Normal tumor marker values & $\mathbf{0 . 0 8 0}$ & 2.17 & $8.75(1.14-67.14)$ \\
Isolated lesions & $\mathbf{0 . 0 6 0}$ & 3.32 & $27.76(1.53-505.17)$ \\
Intervertebral space destruction & $\mathbf{0 . 0 1 0}$ & 3.14 & $23.02(3.07-172.64)$ \\
Paraspinal abscess & 0.997 & - & - \\
Formation of sequestrum & 0.992 & - & - \\
\hline
\end{tabular}

Note: Bold values indicate statistical significance $P<0.1$.

no pain worsens at night, high CRP value, normal tumor marker values, isolated lesions, intervertebral space destruction, paraspinal abscess, and no vertebral appendices involvement were more significant indicators for spinal TB as compared with MT $(\mathrm{P}<0.05)$.

The above-mentioned 10 potential risk factors selected from univariate analysis were subjected to multiple logistic regression analysis, and the results of multivariate analysis are shown in Table 2. Patients with high CRP value and intervertebral space destruction had a significantly higher likelihood of diagnosis as spinal TB $(\mathrm{P}<0.1)$. Meanwhile, the likelihood of diagnosis as spinal TB was significantly decreased in patients with pain worsens at night, elevated tumor marker values, and skip lesions $(\mathrm{P}<0.1)$. In addition, the result of multivariate analysis also showed that age, tumor history, low fever and night sweats, paraspinal abscess, and vertebral appendices involvement were not independent diagnostic factors for differentiating spinal TB and MT.

\section{Development of the Scoring System}

As shown in Table 2 , the regression coefficient $\beta$ values of the independent diagnostic factors in multiple logistic regression model were determined. After rounding off to the nearest integer, the scores of each item in the scoring system were defined as: 2 points for no pain worsens at night and normal tumor marker values; and 3 points for high CRP value, no skip lesions and intervertebral space destruction (Figure 2). The total score was calculated for each patient, and TB cases showed significantly higher total scores than MT cases (mean, 10.3 vs. 4.9, $\mathrm{P}<0.001)$. According to ROC curve, the cut-off value of 7.5 yielded the largest area under ROC with a sensitivity of $88.6 \%$ and specificity of $86.7 \%$ (Figure 3 ). Therefore, 7.5 was selected as the optimal cut-off value of the scoring system.

Herein, as shown in Figure 2, the new scoring system is established with 5 items (pain worsens at night, CRP value, tumor marker values, skip lesions, and intervertebral space destruction). The total score of the system ranges

\begin{tabular}{lc}
\hline Factors & Scores \\
\hline Pain worsens at night & 2 \\
no & 0 \\
$\quad$ yes & \\
CRP value & 0 \\
$\quad$ normal & 3 \\
$\quad$ high & \\
Tumor marker values & 2 \\
$\quad$ normal & 0 \\
$\quad$ high & \\
Skip lesions & 3 \\
$\quad$ no & \\
$\quad$ yes & 0 \\
Intervertebral space destruction & \\
no & 0 \\
$\quad$ yes & 3 \\
\hline
\end{tabular}

Total score

$>7.5 \quad$ Spinal TB

$<7.5 \quad$ Spinal MT

Figure $2 \mathrm{New}$ proposed scoring system for differential diagnosis of spinal TB and MT. 


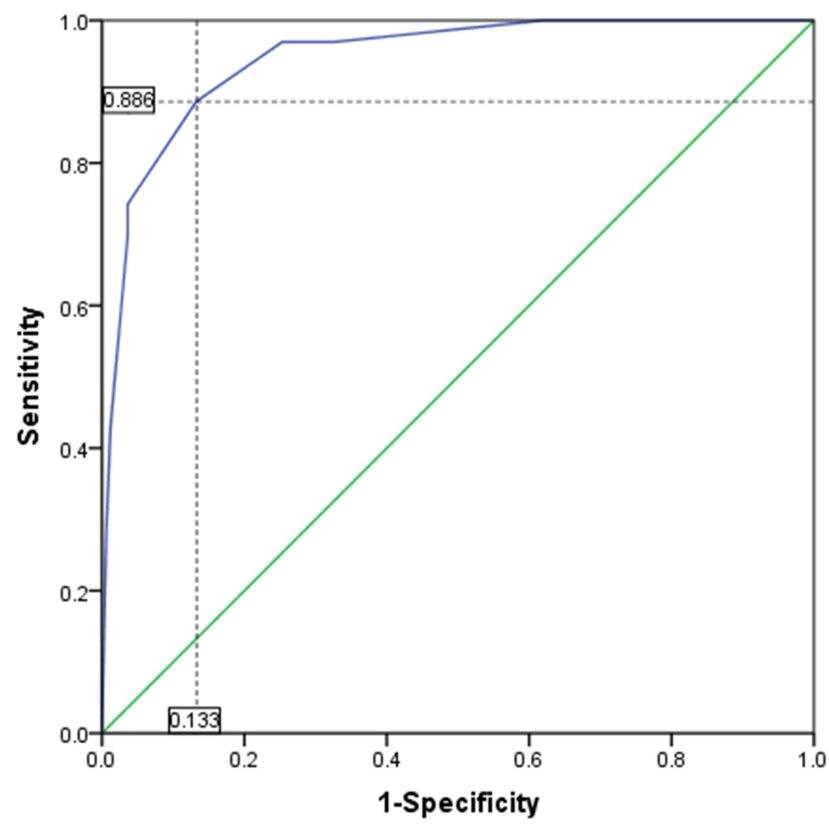

Figure 3 ROC curve analysis to determine the optimal cut-off value of the scoring system.

from 0 to 13 , in which a total score of $>7.5$ indicates a predicted diagnosis of spinal TB, otherwise, spinal MT.

\section{Illustrative Case Presentations and Validation of the Scoring System Case I}

A 56-year-old woman had T6 vertebral lesion causing spinal cord compression. She did not have pain worsens at night (2 points). Laboratory tests showed higher CRP ( 3 points) and tumor marker (AFP) values (0 points). Radiological examination displayed neither skip lesions (3 points) nor intervertebral space destruction ( 0 points). Her total score was 8 , and thus her predicted diagnosis was spinal TB.

\section{Case 2}

A 60-year-old man had T8 and L2 vertebral lesions causing spinal cord compression. He presented with no pain worsens at night (2 points). Laboratory tests showed higher CRP value ( 3 points) and normal tumor marker values (2 points). Radiological examination displayed skip lesions (0 points) without intervertebral space destruction ( 0 points). His total score was 7 , and thus his predicted diagnosis was spinal MT.

The total scores together with their predicted diagnoses of all patients in the validation cohort were calculated using the new proposed scoring system as the above examples demonstrated. The comparisons between the
Table 3 Validation of the Scoring System for Differential Diagnosis of Spinal TB and MT (Validation Cohort)

\begin{tabular}{|l|l|l|}
\hline \multirow{2}{*}{ Total System Score } & \multicolumn{2}{|l|}{ Actual Pathological Diagnosis } \\
\cline { 2 - 3 } & Spinal TB & Spinal MT \\
\hline$>7.5(\mathrm{~TB})$ & $58(87.9 \%)^{*}$ & $7(8.4 \%)$ \\
$<7.5$ (MT) & $8(12.1 \%)$ & $76(91.6 \%)^{* *}$ \\
Total & 66 & 83 \\
\hline
\end{tabular}

Notes: $*$ Sensitivity $=87.9 \%$. $*$ Specificity $=91.6 \%$.

predicted diagnoses and the actual pathological diagnoses revealed that 58 of $66 \mathrm{~TB}$ cases and 76 of 83 MT cases obtained correct prediction (Table 3 ). Therefore, the sensitivity and specificity of the new scoring system for differentiating spinal TB from MT were $87.9 \%$ and $91.6 \%$, respectively.

\section{Discussion}

Spinal TB and spinal MT have similar clinical and imaging presentations, which poses a challenge to clinicians in differential diagnosis, especially for some atypical cases. Although pathological evaluation is the gold standard to confirm the diagnosis, performing biopsies in all patients with spinal cord compression is not feasible due to the urgent need for surgical decompression. Ideally, it would be favorable if surgeons could quickly and accurately make the differential diagnosis with the help of a handy assessment tool. To address this situation, the present study established a novel diagnostic scoring system for discriminating spinal TB from MT. This scoring system was composed of 5 items identified from univariate and multivariate analyses: pain worsens at night ( 0 or 2 points), CRP value (0 or 3 points), tumor marker values ( 0 or 2 points), skip lesions ( 0 or 3 points), and intervertebral space destruction ( 0 or 3 points). Patients who scored higher than 7.5 could be diagnosed as spinal TB, otherwise, spinal MT. According to the internal validation, the sensitivity and specificity of the system were $87.9 \%$ and $91.6 \%$, respectively.

\section{Clinical Factors}

Clinically, back pain is the most common symptom for spinal MT. Typically, the pain often worsens at night owing to distension of the epidural venous plexus and lower endogenous corticoid secretion. ${ }^{15}$ In our study, pain worsens at night was found to be an independent indicator for spinal MT. For spinal TB, the most frequently reported symptoms were also back pain, followed by low fever, body 
weight loss, neurological abnormalities, and night sweats. ${ }^{16}$ In our study, the specific symptoms of TB, i.e., low fever and night sweats, were selected for comparison. Although the result of univariate analysis in our study showed that low fever and night sweats was a factor for differential diagnosis, it failed to achieve a significant difference in multivariate analysis. In addition, age and tumor history were identified to be predictive parameters differentiating tuberculous from malignant pleural effusions, ${ }^{17}$ and they are also commonly used information for differential diagnosis between spinal TB and MT in clinical practice, but our results revealed that neither were regarded as independent diagnostic factors.

\section{Laboratory Factors}

ESR and CRP are generally raised in the majority of patients with active TB. Although they are not unique to TB, the elevation of ESR and CRP is helpful in the diagnosis of spinal TB. ${ }^{18}$ Javed et al suggested that ESR was more sensitive in differentiating spinal TB from tumors than CRP, ${ }^{19}$ while Sudprasert et al proposed that CRP played a better role than ESR in evaluating the response to treatment and prognosis of spinal TB. ${ }^{20}$ In our study, the results showed that CRP rather than ESR was an independent diagnostic factor. Similarly, in patients presenting with lymphocytic exudative pleural effusion, raised pleural fluid CRP level was also an indicator in discriminating between tuberculous pleuritis and malignant pleural effusion. ${ }^{21}$

Clinically, although tumor markers are mainly used to monitor cancer recurrence after treatment, and only a few tumor markers are used for screening (e.g., AFP for hepatocellular carcinoma and PSA for prostate cancer). The increased concentration of a tumor marker is an earlier indicator of MT than clinical presentation and imaging examinations are. ${ }^{22}$ In our study, due to the variety of MTs and limited sample size, a tumor marker combination was used for screening spinal MT. If any of the tumor makers was above the normal range, the patient would be more likely diagnosed as spinal MT.

\section{Radiological Factors}

Imaging tests usually differentiate spinal TB from MT. The typical manifestations of spinal TB are contiguous destroyed vertebral bodies with involvement of intervertebral discs and paravertebral abscesses; while the spinal MT mainly presents as vertebral bone destruction and soft tissue mass with rare disc involvement. ${ }^{3}$ Besides discal changes, a comparative study showed that combined vertebral body and posterior elements involvement, skip lesions, solitary lesion, concentric collapse abscess formation, etc. were MRI features for differentiating the cases of spinal TB and $\mathrm{MT}^{5}$ In our study, skip lesions and intervertebral space destruction were identified to be the two independent diagnostic factors, and included in the scoring system. For other imaging features, take paraspinal abscess as an example, it is a manifestation highly suggestive of spinal TB, but sometimes TB cases are in the developmental stage in which the abscess has not yet formed (high specificity but low sensitivity).

Despite the presence of characteristic imaging findings indicative of spinal TB or MT, there can still be misdiagnosis. For example, an atypical spinal TB case with noncontiguous multiple bone destruction without paravertebral abscess or destruction of adjacent intervertebral discs, ${ }^{8}$ a case of isolated intra-spinal TB without destruction of vertebral body, vertebral arch or intervertebral disc, ${ }^{9}$ and a TB case with the presence of a solitary pulmonary nodule ${ }^{10}$ were all misdiagnosed as spinal MT. Similarly, due to radiological similarities, it is also possible that patients with atypical spinal MT may be erroneously diagnosed and treated for TB. ${ }^{12-14}$ To better differentiate spinal TB from MT, advanced imaging techniques such as CT perfusion and dynamic contrast-enhanced MRI were investigated and showed promising results. ${ }^{3,23}$ However, they were limited for routine clinical use due to cost and time constraints. Moreover, diagnosis of spinal TB or MT should not be based on imaging alone, and the differentiation requires a combination of clinical, laboratory, and radiological aspects. Therefore, this scoring system could prove to be a useful tool for the differential diagnosis. It is comprehensive and practical, yet highly accurate.

There are several limitations to the present study. It is a retrospective study, with cases limited to a single institution. The lack of external validation of the scoring system can be considered as a major flaw. Secondly, to make the scoring system simple and practicable, the laboratory data was dichotomized according to the normal range rather than a more optimal cut-off value determined by ROC curve. In addition, this proposed diagnostic scoring system could just be regarded as an assistant tool, while histological confirmation of the presumed diagnosis by biopsy is always the gold standard.

In conclusion, by systematic analysis of clinical, laboratory, and radiological factors, we identified that pain worsens at night, CRP value, tumor marker values, skip lesions, and intervertebral space destruction were independent factors for differentiating spinal TB from MT. Moreover, a novel 
diagnostic scoring system was developed based on the independent diagnostic factors and validated internally. Further external validation using the new samples and the independent multiple raters is needed before this system could be accepted for general use.

\section{Acknowledgments}

This study is supported by the National Natural Science Foundation of China (Grant No. 81501465). Shuang Cao, Xin Gao, Guangjian Bai, and Baoquan Xin are co-first authors for this study.

\section{Disclosure}

All authors declare that they have no conflicts of interest.

\section{References}

1. Garg RK, Somvanshi DS. Spinal tuberculosis: a review. J Spinal Cord Med. 2011;34(5):440-454. doi:10.1179/2045772311Y.0000000023

2. Spratt DE, Beeler WH, de Moraes FY, et al. An integrated multidisciplinary algorithm for the management of spinal metastases: an international spine oncology consortium report. Lancet Oncol. 2017;18 (12):e720-e730. doi:10.1016/s1470-2045(17)30612-5

3. Lang N, Su M-Y, Yu HJ, Yuan H. Differentiation of tuberculosis and metastatic cancer in the spine using dynamic contrast-enhanced MRI Eur Spine J. 2015;24(8):1729-1737. doi:10.1007/s00586-015-3851-z

4. Jain AK. Tuberculosis of the spine: a fresh look at an old disease. $J$ Bone Joint Surg Br. 2010;92(7):905-913. doi:10.1302/0301620X.92B7.24668

5. Mittal S, Khalid M, Sabir AB, Khalid S. Comparison of magnetic resonance imaging findings between pathologically proven cases of atypical tubercular spine and tumour metastasis: a Retrospective Study in 40 patients. Asian Spine J. 2016;10(4):734-743. doi:10.4184/ asj.2016.10.4.734

6. Kumaran SP, Thippeswamy PB, Reddy BN, Neelakantan S, Viswamitra S. An institutional review of tuberculosis spine mimics on MR imaging: cases of mistaken identity. Neurol India. 2019;67 (6):1408-1418. doi:10.4103/0028-3886.273630

7. Wang LN, Wang L, Liu LM, Song YM, Li Y, Liu H. Atypical spinal tuberculosis involved noncontiguous multiple segments: case series report with literature review. Medicine. 2017;96(14):e6559. doi:10.1097/MD.0000000000006559

8. Meng Y, Gong Q, Liu H. A case of atypical spinal tuberculosis mimicking metastatic tumor. Spine J. 2016;16(4):e267-e270. doi:10.1016/j.spinee.2015.10.055

Infection and Drug Resistance

\section{Publish your work in this journal}

Infection and Drug Resistance is an international, peer-reviewed openaccess journal that focuses on the optimal treatment of infection (bacterial, fungal and viral) and the development and institution of preventive strategies to minimize the development and spread of resistance. The journal is specifically concerned with the epidemiology of
9. Yu Y, Wang X, Du B, Yuan W, Ni B, Chen D. Isolated atypical spinal tuberculosis mistaken for neoplasia: case report and literature review. Eur Spine J. 2012;22(S3):302-305. doi:10.1007/s00586-012-2294-z

10. Ringshausen FC, Tannapfel A, Nicolas V, et al. A fatal case of spinal tuberculosis mistaken for metastatic lung cancer: recalling ancient pott's disease. Ann Clin Microbiol Antimicrob. 2009;8(1):32. doi:10.1186/1476-0711-8-32

11. Sarangapani A, Fallah A, Provias J, Jha NK. Atypical presentation of spinal tuberculosis. Can J Surg. 2008;51(6):E121.

12. Kessler RA, Steinberger J, Chen S, Baron R, Caridi JM. Lung adenocarcinoma presumed to be pott's disease in a 28 -year-old patient: a case report and review of literature. Surg Neurol Int. 2019;10:208. doi:10.25259/SNI_403_2019

13. Flais J, Coiffier G, Brillet E, Perdriger A, Guggenbuhl P. Atypical presentation of spine bone metastasis in prostate cancer mimicking pott's disease. Clin Cases Miner Bone Metab. 2017;14(2):239-240. doi:10.11138/ccmbm/2017.14.1.239

14. Zheng C-Y, Liu D-X, Luo S-W, Du S-X. Imaging presentation highly manifested as tuberculosis in a case of spinal metastatic carcinoma. Orthopedics. 2011;34:e436-e438. doi:10.3928/01477447-2011062732

15. Yanez ML, Miller JJ, Batchelor TT. Diagnosis and treatment of epidural metastases. Cancer. 2017;123(7):1106-1114. doi:10.1002/ cncr.30521

16. Chen CH, Chen YM, Lee CW, Chang YJ, Cheng CY, Hung JK. Early diagnosis of spinal tuberculosis. J Formos Med Assoc. 2016;115 (10):825-836. doi:10.1016/j.jfma.2016.07.001

17. Porcel JM, Vives M. Differentiating tuberculous from malignant pleural effusions: a scoring model. Med Sci Monit. 2003;9(5): CR175-CR180.

18. Wang B, Gao W, Hao D. Current study of the detection and treatment targets of spinal tuberculosis. Curr Drug Targets. 2020;21 (4):320-327. doi:10.2174/1389450120666191002151637

19. Javed G, Laghari AA, Ahmed SI, et al. Development of criteria highly suggestive of spinal tuberculosis. World Neurosurg. 2018;116:e1002-e1006. doi:10.1016/j.wneu.2018.05.149

20. Sudprasert W, Piyapromdee U, Lewsirirat S. Neurological recovery determined by $\mathrm{C}$-reactive protein, erythrocyte sedimentation rate and two different posterior decompressive surgical procedures: a Retrospective Clinical Study of patients with spinal tuberculosis. J Med Assoc Thai. 2015;98(10):993-1000.

21. Chierakul N, Kanitsap A, Chaiprasert A, Viriyataveekul R. A simple C-reactive protein measurement for the differentiation between tuberculous and malignant pleural effusion. Respirology. 2004;9(1):66-69. doi:10.1111/j.1440-1843.2003.00521.x

22. Solnica B. Not easy to become a tumor marker. Pol Arch Med Wewn. 2016;126(11):845-846. doi:10.20452/pamw.3699

23. Shankar J, Jayakumar P, Vasudev M, Ravishankar S, Sinha N. The usefulness of CT perfusion in differentiation between neoplastic and tuberculous disease of the spine. $J$ Neuroimaging. 2009;19 (2):132-138. doi:10.1111/j.1552-6569.2008.00265.x antibiotic resistance and the mechanisms of resistance development and diffusion in both hospitals and the community. The manuscript management system is completely online and includes a very quick and fair peerreview system, which is all easy to use. Visit http://www.dovepress.com/ testimonials.php to read real quotes from published authors. 